\title{
Far-Infrared Detection
}

\author{
Maurice Kimmitt, Colchester
}

(University of Essex)

"Thermometer No. 1 rose, in 16 minutes, $8 \frac{3}{4}$ degrees when its centre was $1 / 2$ inch out of the visible rays." With these words Sir William Herschel announced the discovery of what we now call the infrared region of the spectrum. The 18th century was then just closing and within a hundred years wavelengths from thermal sources extending to 150 micrometres had been isolated and measured. Meanwhile Clark Maxwell had produced his theory of electromagnetic radiation and Hertz was carrying out his famous experiments on the properties of these waves - experiments which included reflection, refraction, polarisation and interference. The gap between infrared and radio waves was closed in the 1920 s and nowadays the generation of coherent waves, using microwave radio techniques, has been extended to less than $0.5 \mathrm{~mm}(500 \mu \mathrm{m})$ and infrared spectroscopy with thermal sources is routinely performed to beyond $2 \mathrm{~mm}$. The name for these wavelengths varies, often dependent on whether the technology employed is optical or microwave. 'Far infrared' is from $\cong 50$ to beyond $1000 \mu \mathrm{m}$ : 'submillimetre' $\cong 100-1000$ $\mu \mathrm{m}$ and 'near-millimetre' means near 1 $\mathrm{mm}$ or $\cong 0.3-3 \mathrm{~mm}$.

William Herschel used a mercury in glass thermometer with a blackened bulb for his initial experiments. His son John discovered absorption bands in the infrared by soaking a piece of black paper in alcohol and placing it in a dispersal spectrum of the Sun. Differential evaporation of the alcohol showed light patches where the radiation was most intense and he showed that the solar infrared region consisted of three or more separate bands. We now know that the

Fig. 1 - Photoconductive detector processes.

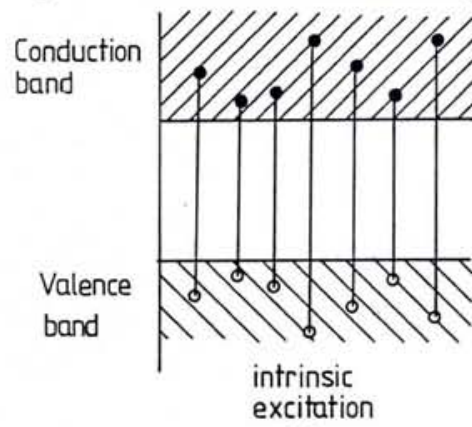

(a)

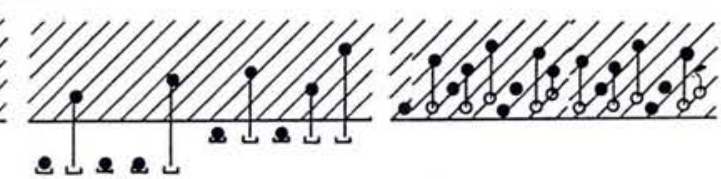

-

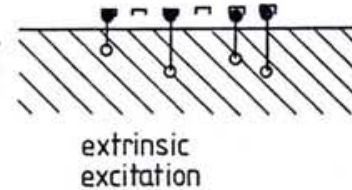

(b) intervening wavelengths are lost due to absorption by water and carbon dioxide in the atmosphere. Atmospheric absorption becomes more intense at longer wavelengths and between 25 and 650 $\mu \mathrm{m}$ the atmosphere is essentially opaque except for a very few high altitude 'windows'. One of the prime motivations for better long wavelength infrared detectors has come from astronomy to cover the gap between conventional infrared wavelength $(<20 \mu \mathrm{m})$ and radioastronomy. Detectors operating in the range $100 \mu \mathrm{m}$ to $2 \mathrm{~mm}$ are also required for plasma diagnostics, for measurement of the very small energy gaps in superconductors, as well as to study the output of a variety of new sources such as free electron lasers and gyrotrons.

\section{Detection Processes}

Early detectors of infrared radiation were thermal devices; the incident energy produced a change in volume of a liquid or a gas and a sophisticated gas thermometer - the Golay detector is still used today. Most thermal detectors rely on the heating of the lattice to produce a subsequent change in an electronic property of a solid. For example, in a thermocouple or thermopile an EMF is produced directly, while in a bolometer the heating results in a change of resistance. The advantage of such detectors is that as long as they absorb the radiation, either directly or via an absorbing surface layer, they are not wavelength-dependent. One of the most widely used detectors for astronomy in the region near to $1 \mathrm{~mm}$ wavelength is a bolometer cooled to about $1 \mathrm{~K}$. A particularly useful detector exploits the pyro- electric effect in which the absorbed energy produces a change of charge.

The most important infrared detectors utilise the energy of absorbed photons directly to produce an electronic change. Infrared detectors rely on internal photoeffects with the energy of the photon raising an electron from a non-conducting to a conducting state.

The relationship between the energy and wavelength of light is

$$
E=1.2 / \lambda
$$

where $E$ is in electron volts and $\lambda$ in micrometres. The existence of a long wavelength limit beyond which the energy of the photon is less than that required to produce the required transition is a characteristic of photon detectors and it is apparent from Eq. (1) that very narrow energy gaps are required for long wavelength detection. Intrinsic photoconduction, illustrated in Fig. 1(a), where the incident photon produces an electron-hole pair is restricted to wavelengths less than $7 \mu \mathrm{m}$ in the normally available semiconductors, but with alloys such as $\mathrm{HgCdTe}$ the range can be extended to about $30 \mu \mathrm{m}$. For longer wavelengths, extrinsic photoconduction is available. By adding an appropriate impurity to a semiconductor, donor or acceptor sites can be produced and, as shown in Fig. 1 (b), the effective energy is that between the impurity level and the conduction or valence band.

In a high mobility semiconductor, such as $n$-InSb cooled to liquid helium temperature, electrons in the conduction band are not well coupled to the lattice. At wavelengths longer than 150 $\mu \mathrm{m}$, these electrons absorb radiation and are heated above the temperatures of the lattice, Fig. 1(c). This increases their mobility and reduces the detector resistance. Not surprisingly, for a wavelength region that extends from the infrared into the short microwave region, at the same time as optical type detectors have been stretched to longer wavelengths, radio type detectors have been employed at shorter wavelengths. These respond to the electrical currents induced in them at the frequency of the radiation, in the same way as a rectifier acts as a nonlinear component in a low frequency electrical circuit. The problem of extending such devices to shorter 
wavelengths is that at the corresponding higher frequencies $\left(300 \mu \mathrm{m}=10^{12}\right.$ $\mathrm{Hz}$ ) capacitance and inductance must be kept very small. Even so considerable success has been achieved with metalsemiconductor (Schottky) diodes, Josephson junctions and, more recently, with superconductor-insulator-superconductor (SIS) diodes.

\section{Detector Assessment}

Complete assessment of any detector is quite complex but for most purposes four parameters are sufficient:

1) wavelength range over which it responds;

2) speed of response;

3) noise equivalent power;

4) area.

The first two parameters are self-explanatory. As pointed out previously, thermal detectors will often perform over a wide range of wavelengths while photodetectors have a cut-off at some long wave limit. Because thermal detectors require the heating of the lattice, they are usually quite slow, typically taking $1 \mathrm{~ms}-1 \mathrm{~s}$ to respond. Photodetectors are fast, typically varying between $1 \mathrm{~ns}$ and $10 \mu \mathrm{s}$.

The noise equivalent power (NEP) indicates the sensitivity of a detector. It is the power required to give a $1: 1$ signalto-noise ratio. As the fundamental sources of noise produce fluctuations in the detector output which increase as the square root of the electrical bandwidth in the detection system, the units of NEP are $\mathrm{W} \mathrm{Hz}^{-1 / 2}$. The area, $A$, is important because larger detectors are often more noisy. In an ideal infrared detector the limiting noise is produced by statistical fluctuations of radiation from the background. In these circumstances, noise increases as the square root of the detector area and this has led to a useful comparison criterion known as the 'specific detectivity' with the symbol $D^{*}$. Its relationship to the NEP is

$$
D^{*}=(A f)^{1 / 2} / \mathrm{NEP}
$$

and it has the units $W^{-1} \mathrm{~Hz}^{1 / 2} \mathrm{~cm}$. One obvious advantage is that $D^{*}$ gets bigger as the detector gets better.

Another useful detector parameter is the responsivity, $R$, usually expressed in V/W. It is related to the NEP by

$$
R=V_{n} / \text { NEP }
$$

where $V_{n}$ is the RMS voltage produced by all noise sources. In a photodetector it is the arrival of a photon that produces some effect and the number of 'photons per watt' increases linearly with wavelength which leads to a response that rises until cut-off at the long wavelength limit. The ideal responses of thermal and photodetectors is illustrated in Fig. 2.

\section{Cooled Detectors}

Although long wavelength thermal detectors operating at room temperature are available, cooling produces dramatic improvements in detectivity. Fig. 3 is a schematic arrangement of a bolometer with 'chopped' incident radiation falling on it. With the electrical circuit shown, an AC signal output will be produced with its amplitude proportional to the change of resistance of the detector. It is immediately obvious that the bolometer should have a small 'thermal mass' and a large temperature coefficient of resistance. A low heat conductance to the 'heat sink' will give improved responsivity but at the expense of a slower response time. By cooling bolometers to $1 \mathrm{~K}$ or less, the specific heat is reduced and the coefficient of resistance increased, both by several orders of magnitude. However, unless the detector is shielded from room temperature background radiation, its NEP will not be significantly improved because the fluctuations from this source will produce a correspondingly increased voltage response. These fluctuations produce, for a $1 \mathrm{~cm}^{2}$ area detector with a $1 \mathrm{~Hz}$ bandwidth, a limiting NEP of 3.54 $\times 10^{-17} T^{5 / 2}$ where $T$ is the temperature of the background in degrees Kelvin. The potential improvement available is dramatic:

Background Temp. (K) Limiting NEP $\left(\mathrm{W} \mathrm{Hz}^{-1 / 2}\right)$

$\begin{array}{rl}300 & 5.5 \times 10^{-11} \\ 77 & 1.8 \times 10^{-12} \\ 4 & 1.1 \times 10^{-15} \\ 0.3 & 1.7 \times 10^{-18}\end{array}$

As this fundamental noise limit is reduced, increased care must be taken to reduce other noise sources. There must also, of course, be an aperture to let the wanted radiation through! This aperture can, however, be very small, particularly in infrared astronomy, and cooled filters which only let through the wanted wavelengths - and consequently only limited background - can also be included. The best NEP achieved with a germanium bolometer at $0.1 \mathrm{~K}$ is about $4.5 \times 10^{-17} \mathrm{~W} \mathrm{~Hz}^{-1 / 2}$, whereas laboratory detectors for spectroscopy in the $0.1-2 \mathrm{~mm}$ range operating at $\cong 1.2 \mathrm{~K}$ have NEPs of $10^{-12}-10^{-13} \mathrm{~W} \mathrm{~Hz}^{-1 / 2}$.

Uncooled photodetectors will not operate at long wavelengths. The reason for this can be understood by reference to Fig. 1. As the energy gap is reduced, thermal background radiation will excite the electrons and fewer will be available when the wanted radiation falls on the device. In practice, detectors for greater than $20 \mu \mathrm{m}$ wavelength are cooled to $4 \mathrm{~K}$ or less. A particularly useful photoconductor is $\mathrm{Ge}: \mathrm{Ga}$ made from very pure

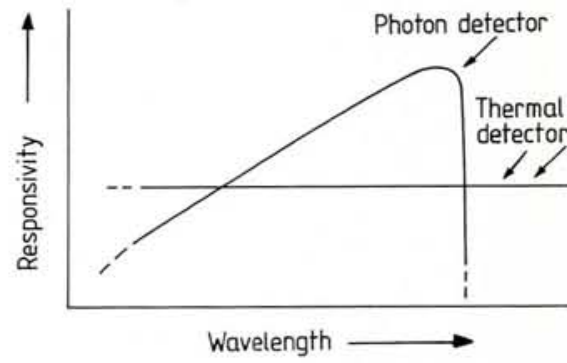

Fig. 2 - Ideal spectral response of thermal and photon detectors.

germanium with about $10^{14}$ gallium atoms $/ \mathrm{cm}^{3}$ added. It has a wavelength range of $30-120 \mu \mathrm{m}$ and when used with a small field of view, NEPs close to $10^{-17} \mathrm{~W} \mathrm{~Hz}^{-1 / 2}$ have been achieved; detectors for more general purpose applications have NEPs of $\cong 10^{-12} \mathrm{~W} \mathrm{~Hz}^{-1 / 2}$. A Ge:Ga detector was included in the very successful IRAS satellite. By applying stress to $\mathrm{Ge}: \mathrm{G}$ a the binding energy of the electrons is reduced and the wavelength range can be extended to 200 $\mu \mathrm{m}$. Shallow impurity levels in GaAs extends the intrinsic detection to about $350 \mu \mathrm{m}$, with electron bolometers operating out to several millimetres.

\section{Heterodyne Detection}

At broadcast and microwave frequencies, the wanted signal radiation is combined with a signal from a 'local oscillator' (LO) and the difference or 'intermediate frequency' (IF) is amplified. There are two fundamental requirements in such a system. First, the detector or 'mixer' in which the signals are combined must be fast enough to respond to the difference frequency. Second, the signal and LO must be spatially coherent; in practice this means that the detector has antenna properties that

Fig. 3 - Schematic arrangement of a cooled bolometer.

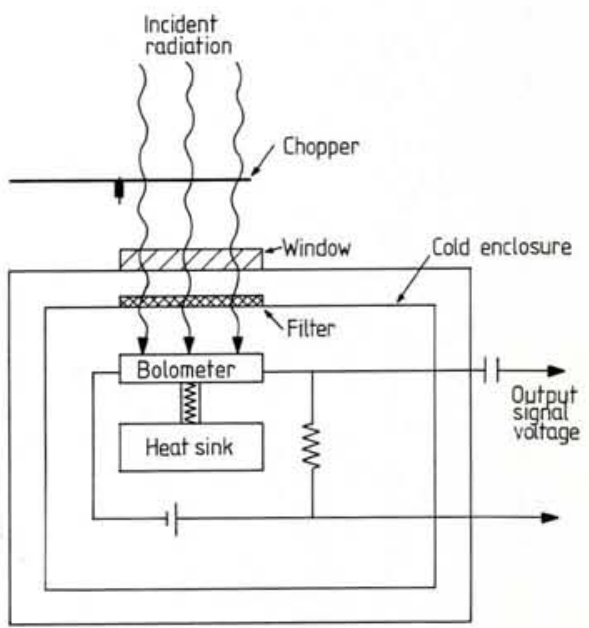


respond to a single mode of the signal field only. The first requirement indicates that heterodyne detection will be particularly useful for narrow line sources, so long as a suitable LO is available with a wavelength output very close to that of the source. The second requirement appears rather restrictive but, particularly in astronomical applications, where the solid angle from the source is small, it can be advantageous. Very sophisticated heterodyne detection systems have been designed for the study of the rotational line emission of molecules such as carbon monoxide, which has been identified in many astronomical objects. Heterodyne detection is also used to measure the Doppler shift of long wavelength lasers used in scattering experiments from plasmas in tokomaks and similar machines.

With the proviso that the mixing must be done efficiently, heterodyne detection offers significant signal-to-noise advantages over normal broad band (video) detectors. The fundamental reason is that the heterodyne system 'sees' background noise only in the frequency band of detection, which is normally limited by the bandwidth of the IF amplifier. This leads to a theoretical $300 \mathrm{~K}$ background-limited 'noise temperature' (q.v.) of less than $10 \mathrm{~K}$ for a $1 \mathrm{~Hz}$ band width.

InSb electron bolometers have been used very successfully as heterodyne detectors at wavelengths near to $1 \mathrm{~mm}$ with noise temperatures of $\cong 300 \mathrm{~K}$. However, these detectors have a relatively slow response time and this limits the IF bandwidth to about $1 \mathrm{M} \mathrm{Hz}$. Extension of microwave technology has produced a number of rectifying diodes for use in the near-millimetre region and two types have emerged in practical receivers. The first of these, Schottky diodes, are metal-semiconductor rectifying junctions. Noise temperatures of a few hundred degrees have been reported in the short millimetre region, rising to more than ten thousand degrees at the shortest wavelengths of operation $\cong 150 \mu \mathrm{m}$. These detectors require quite high LO power, typically a few $\mathrm{mW}$, and at far-infrared wavelengths the only available sources are lasers. The most useful of these are of the opticallypumped type where a $\mathrm{CO}_{2}$ laser at 10 $\mu \mathrm{m}$ is used to invert a specific rotational level in a second laser which then radiates in the far-infrared. The disadvantages of such laser local oscillators is that they are large, often several metres in length, and are non-tunable. However, for astronomical purposes it is often possible to find a laser line close enough to a particular molecular emission for mixing to be possible and Fig. 4 illustrates the type of arrangement used with great success by H.P. Röser and his colleagues at the Max Planck Institute of Radioastronomy in Bonn. They have designed systems for use at high altitude sites and in airborne observatories.

An interesting aspect of heterodyne detection at these wavelengths is that it is difficult to make very small dimension waveguides, so optics are used to combine the signal and local oscillator beams, and to couple to the detector. However, to avoid interference effects these optical systems must be very complex and this has led to a new type of design called 'quasi-optics'. Put simply, 'quasi-optics' represents the open path equivalent of microwave circuit elements. Fig. 4 is an example of how the combined beams can be coupled into the very small area of a Schottky diode.

The second type of diode which is now being increasingly employed for long-wavelength mixing is made from superconductor-insulator-superconductor (SIS) structures. These have largely superseded detectors using Josephson junctions, which have also been used with considerable success. SIS structures show non-linearities due to 'quasiparticle' tunnelling through the insulating barrier and a full quantum mechanical treatment finds that they are not only inherently low-noise devices but that, unlike classical mixers, they are capable of giving gain. This is important because it means that the overall system noise will be reduced. These diodes were originally used at millimetre wavelengths but have now been extended well into the sub-millimetre region. Compared with Schottky diodes there is the disadvantage that superconducting detectors must be cooled to below their critical temperature, but this has the benefit that, as long as the background is also cooled, very low noise temperatures are possible; $\cong 100 \mathrm{~K}$ has been achieved at $1 \mathrm{~mm}$. A particularly important feature is that very small LO powers of, typically, $10^{-8} \mathrm{~W}$ are required and these can be provided by using klystrons and harmonic generators. As the klystron frequency can be varied by about $10 \%$, the heterodyne receiver can be tuned to a specific wavelength.

\section{Other Developments}

Semiconductor technology is developing rapidly and a number of the new structures show promise. Mixers using $\mathrm{GaAs} / \mathrm{GaAlAs}$ resonant tunnelling devices and detectors using superlattice technology have been operated at sub-

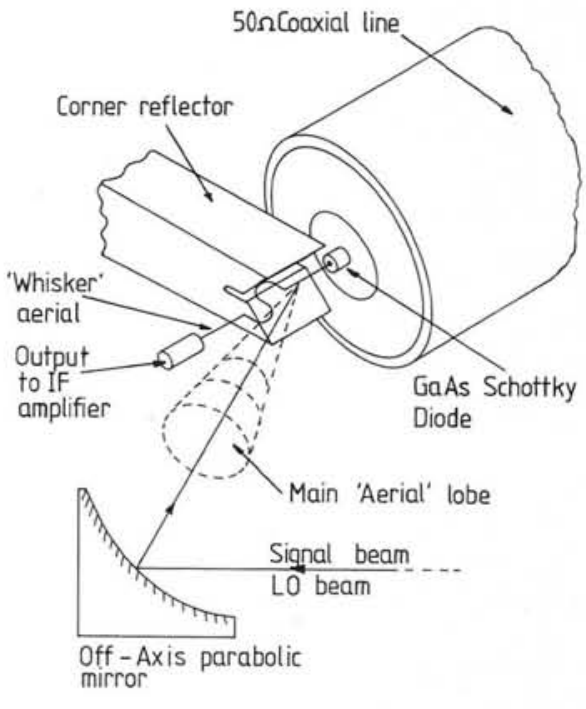

Fig. 4 - Schottky diode quasi-optical mixer.

millimetre wavelengths. Detection due to transitions between Rydberg states of alkali metal vapours has been demonstrated. These have energy level spacings corresponding to long wavelengths. Excellent NEPs have been achieved but clearly such detectors are less convenient than solid state ones.

While the astronomical community has led a steady development of long wavelength detection there are other requirements, particularly in the study of very fast pulsed sources. Pyroelectric detectors are available with sub-nanosecond response times but low detectivity. By suitable 'doping', Ge:Ga detectors combining $1 \mathrm{~ns}$ response and a good NEP can be made and a faster electron bolometer using $\mathrm{HgCdTe}$ has been developed by the author and his colleagues. Superconducting junctions and Schottky diodes can also be used as fast pulse detectors. The 'photon drag' effect (where the momentum of the radiation is transferred to free electrons in a semiconductor) provides a fast and convenient room temperature detector for high power sources.

Far-infrared science and engineering has had a long and fascinating history and it is clear that continuing detector development will ensure an exciting future.

\section{FURTHER READING}

Chantry G.W., Long-Wave Optics, Vol. 1: "Principles", (Academic Press, London) 1984.

Infrared and Millimeter Waves, Vol. 3 "Submillimeter Techniques", Ed. K.J. Button, (Academic Press, New York) 1980.

Guenther B.D. and Kruse P.W., "Submillimeter Wave Detector Workshop", Int. J. Infra. and Millimeter Waves 7 (1986) 1091-1109. 